\title{
Learning Analytics Architecture to Scaffold Learning Experience through Technology-based Methods
}

\author{
Jannicke Baalsrud Hauge ${ }^{1 *}$, loana Stanescu ${ }^{2}$, Sylvester Arnab ${ }^{3}$, Pablo Moreno \\ Ger $^{4}$, Theodore Lim ${ }^{5}$, Angel Serrano-Laguna ${ }^{4}$, Petros Lameras ${ }^{6}$, Maurice Hendrix ${ }^{7}$, \\ Kristian Kiili ${ }^{8}$, Manuel Ninaus ${ }^{9}$, Sara de Freitas ${ }^{10}$, Alessandro Mazzetti ${ }^{11}$, Anders \\ Dahlbom $^{12}$, Cristiana Degano ${ }^{11}$ \\ 1, *University of Bremen, Bremer Institut für Produktion und Logistik, Germany, \\ jmbh@uni-bremen.de \\ 2"Carol I" National Defence University, Romania, ioana.stanescu@aldnet.ro \\ ${ }^{3}$ Disruptive Media Learning Lab, Coventry University, UK; s.arnab@coventry.ac.uk \\ ${ }^{4}$ Universidad Complutense de Madrid, Spain,\{angel.serrano;pablom\}@fdi.ucm.es \\ ${ }^{5}$ Herriot-Watt University, UK, T.Lim@hw.ac.uk \\ ${ }^{6}$ Serious Games Institute, Coventry University, UK, PLameras@cad.coventry.ac.uk \\ ${ }^{7}$ School of Science and Technology, University of Northampton, UK, \\ Maurice.hendrix@northampton.ac.uk \\ ${ }^{8}$ Tampere University of Technology, Finland, kristian.kiili@tut.fi \\ 9University of Graz, Austria, manuel.ninaus@uni-graz.at \\ ${ }^{10}$ Curtin University, Australia, Sara.deFreitas@curtin.edu.au \\ ${ }^{11}$ Gruppo SIGLA S.r.I, Italy, \{alessandro.mazzetti, cristiana.degano\}@grupposigla.it \\ ${ }^{12}$ Högskolan i Skövde; Box 408, 54128 Skövde, Sweden, anders.dahlbom@his.se
}

\begin{abstract}
The challenge of delivering personalized learning experiences is often increased by the size of classrooms and online learning communities. Serious Games (SGs) are increasingly recognized for their potential to improve education. However, the issues related to their development and their level of effectiveness can be seriously affected when brought too rapidly into growing online learning communities. Deeper insights into how the students are playing is needed to deliver a comprehensive and intelligent learning framework that facilitates better understanding of learners' knowledge, effective assessment of their progress and continuous evaluation and optimization of the environments in which they learn. This paper discusses current SOTA and aims to explore the potential in the use of games and learning analytics towards scaffolding and supporting teaching and learning experience. The conceptual model (ecosystem and architecture) discussed in this paper aims to highlight the key considerations that may advance the current state of learning analytics, adaptive learning and SGs, by leveraging SGs as an suitable medium for gathering data and performing adaptations.
\end{abstract}

Keywords: game mechanics; GLEANER; personalization

\section{Introduction}

The EU Education and Training 2020 [1] identified that a major challenge for improving the quality and efficiency of formal education (K12) is to ensure the acquisition of 21st century skills as means of developing excellence for allowing Europe to retain a strong global role. The 21st century heralds a new beginning of digital technologies, which offers opportunities to improve quality, access and equity in education and training. 
To effectively support the acquisition of 21 st century skills, both teachers and students need to receive better and more personalized support in conjunction with effective deployment of technology-assisted approaches. Evidence $[2,3,4,5]$ shows the emphasis must be on how students can apply skills and knowledge rather than just knowledge proficiency. This presents a technical challenge in identifying the right parameters to monitor and assess the quality of the learning process and experience related to students' progress.

The quality and efficacy of a learning process is influenced by the quality of teaching, learning support and environment. How can learners' needs and performance be effectively monitored and supported? The Vygotskian scaffolding concept [6, 7] offers a potential basis. This concept allows individual learners to be given tailored support during the learning process, personalized to their individual needs. Understanding how the different learning preferences influence how learners learn most efficiently $[8,9]$ is essential to maximizing the impact of a teaching and learning process.

The scaffolding support required may be different for each individual, and may depend on several factors such as motivation, prior knowledge, psycho-physiological needs, interest, and context. With respect to the need for personalized support, the growing number of learners, e.g. students/trainees in classes and on Continuing Professional Development (CPD), presents a new challenge in terms of a more scalable and sustainable ways of monitoring learners performances as well as teaching strategies and methods. Each learner will need personalized feedback based on their learning advances. Consequently, the complexity in the monitoring and tracking of different factors increases. Timely access to selected, actionable information gathered across diverse data sources is hence a key determinant for scaffolding success and personalizing the learner's experience in real-time.

Early analytic techniques currently implemented in education include attention metadata [10]; social network analysis [11] and recommender systems [12]. In terms of adapting to user models, adaptive web systems incorporate user models to adapt the systems' behavior to individual users [13]. Intelligent tutoring systems (e.g., [14]) aim to offer students individualized practice, as well as the dynamic composition and delivery of personalized learning utilizing reusable learning objects.

Beyond these learning analytics tools few capture variations of data on the actual learning process a learner will go through. Learning analytics-specific approaches are still at their infancy [15]. Another challenge is therefore how to best capture and reason these big datasets and utilize the analysis to inform teaching approaches for the individual learners and adapt to their experience, be it in a virtual learning environment or the actual physical classroom delivery/deployment.

In this perspective, this paper first looks into the potential of learning analytics in SG as well as how to process big data for learning analytics, thereafter it introduces a conceptual model that can support the scaffolding of teaching and learning experience within a formal setting. This model consists of four (4) components- (i) learning analytics platform, (ii) in-game and stealth measures, (iii) user modelling, adaptive control and visual analytics, and (iv) map of pedagogical patterns to game mechanics. Based upon this model, it describes a conceptual ecosystem including the presentation of results based on studies carried out under the EU-Funded Games and Learning Alliance (GALA) using the learning analytic framework called GLEANER.

The last section concludes the paper with the future research and development requirements for implementing the system and architecture in practice.

\section{The potential of learning analytics in SG}

The Learning Analytics discipline [16, 17] consolidates its impact within Technology Enhanced Learning (TEL) as a methodology to determine how data collected from learners can help to improve the many aspects of the educational process. Despite being a relatively new discipline, research on Learning Analytics has shown promising benefits. Solutions such as LOCO-Analyst and SNAPP [17] focus on the interactions performed by the students within the Learning Management Systems (LMSs), whose contents are mainly based on static learning objects with low user interactivity such as documents, presentations or videos. However, there are other educational resources, such as simulations games that present a higher level of interactivity and engagement and, therefore, generate a higher and more varied amount of log data to be analyzed.

Gaming naturally taps into our desire to be entertained, promoting sustained engagement. A gamebased approach to monitor learning can provide insights into the learning experience, the learning environment and the actual performance during game play. Technologies developed for serious/nonentertainment games have become increasingly popular capitalizing on the engaging factor of the game mechanics. Games such as America's Army or Code of Everand reach large numbers of 
players, engaging them for long periods of time. Not only does this make them attractive platforms for learning and training, but also they provide an opportunity for essential educational data to be recorded, monitored and analyzed [18].

It is anticipated that over the next two to three years, there will be a significant drive to encapsulate game-based learning and learning analytics to support scaffolded learning as new technologies are adopted [19].

The system-generated data would aid educationalists on identifying student's learning problems or concerns and thereby offering a customized learning experience. Both quantitative and qualitative analysis will be required to unpack conceptions, beliefs and actions of learning instances and associate them with the teaching practice [20]. This would include a realistic and valued interpretation of both efficient and inefficient experiences of the environment and offering suggestions for progressing from one end to the other.

\subsection{Learning analytics and personalized learning}

Learning analytics is key to improving learning, but also to provide learners with personalized recommendations in their learning based on previous learning activity orchestrations [21]. This would offer a change in the realm of learning analytics because it would offer not only academic management but also would provide computer-generated suggestions on learning content that is directly related to student's preferences and needs.

This sets the ground for exploratory research on how learning analytics support personalized learning experiences through customized recommendations. The accuracy of such recommendations would be carefully considered possibly through integrating data from multiple sources for improving the accuracy of a student profile and subsequent personalization of content. Therefore, the inclusion of data from other sources such as mobile devices, physical data from supervision meetings and game environments in conjunction with the use of university resources such as libraries as well as learners' preferences might result in a more complete learner profile.

\subsection{Learning analytics and assessment}

While games promise to teach in innovative ways, the assessment of their effectiveness tends to gravitate back to written examinations or debriefing sessions due to the lack an appropriate assessment means to generate rigorous and reliable student results [22, 23, 24]. This has caused an increasing gap between the purportedly deep learning that can be conveyed by educational games and the shallow techniques that are used to assess learners' performance at present.

Different solutions and techniques have been proposed to fill this gap, including using the games themselves to assess the performance [25]. Intrinsic techniques leverage the game technology itself to create in-game assessment tools, either explicit (asking in-game questions, having the player perform a specific evaluated task, etc.) or implicit (producing a grade through indirect measurement of performance). The field of implicit in-game evaluation (also called stealth assessment [25, 26, 27]) offers rich opportunities: since games are highly interactive and complex software artefacts, they can produce a great amount of data on how the user is playing, which is readily available for assessment purposes. However, the best methods and techniques for utilizing all this data to measure learning effectiveness and assessing learners most effectively remains an open research and development challenge.

In addition, according to cognitive psychology of learning, our thinking is based on conceptual representations of our experiences and complex relations between these concepts and experiences. This kind of modelling makes it possible to reproduce conceptual learning processes and thus uncover the frequencies, dependencies and patterns behind the conceptual change and learning transfer. When adding a Big Data dimension, the national level learning challenges can be uncovered and national curriculum designers will benefit from important real-time information [28]. It is therefore useful to scope the current applications and refinements of Learning Analytics techniques, adapting them to the specific context of SGs in order to leverage the vast amount of trace data that games can generate to provide rich learner-based assessment information. The following sections 3 and 4 discuss a conceptual architecture for optimizing the use of Learning Analytics and SGs towards supporting the dynamic nature of teaching and learning processes. 


\section{The conceptual architecture}

To analyze learning within a game environment, the analytics platform needs to take into considerations the use of stealth and in-game measures influenced by the pedagogical perspectives of the intended game play towards understanding the learners and providing personalized feedback and support to them. In this perspective, a general architecture and the considerations based on existing work in the area is discussed in this section. The discussion includes existing and on-going studies supporting the components highlighted in fig. 1 (b), supporting the iterative analytic process illustrated in fig. 1(a). The framework and its realization are described further on.

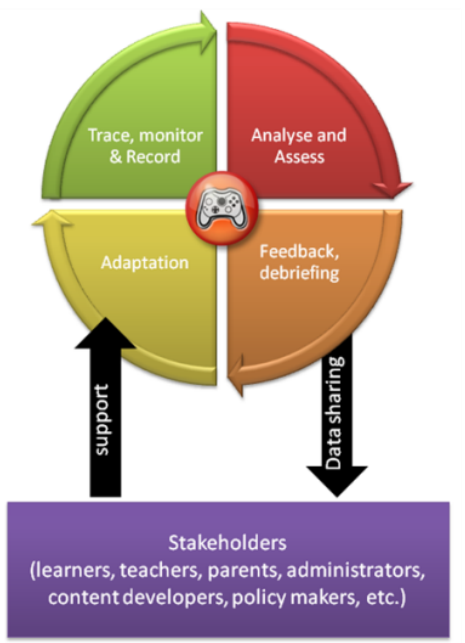

(a)

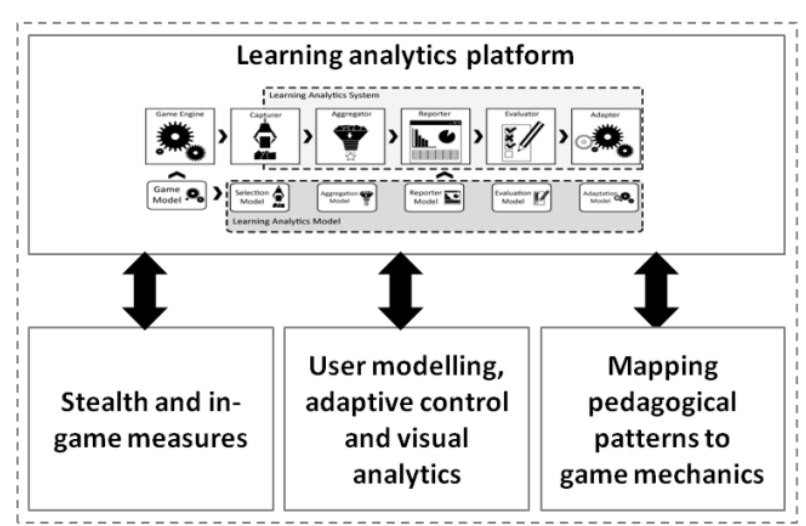

(b)

Figure 1. (a) The iterative and continuous process of monitoring and analyzing data based on the metrics and KPIs specific to the stakeholders, supported by (b) the key technical components to support a pedagogically-driven learning analytics and adaptive platform

It is important to understand learners' preferences to allow learning and playing patterns to be analyzed and adapted. Adaptivity to different learning preferences and needs requires conceptualizing the user in terms of their learning capabilities and learning progress by investigating what parameters would most accurately characterize the learners - and how these map to specific learner categories.

Capturing learners' activity during game play can help measure these parameters. However, meaningful interaction and engagement from a learning perspective need to be defined and, correctly and ethically captured (using opt in and opt out user consent) during game-play. In-game measures have to be based on the theoretical construct structured by the pedagogical patterns related to game design patterns, which provides the relationship between game and learning contents. This provides a more effective way to data mine relevant data, extract meaningful learning interactions and gain insight on how learning can be scaffolded. Analysis from this real-time data can be used to build the relevant user models, which could be included in the feedback, reporting and adaption process. The visualization of the analyzed data is also important to maximize the benefits of the correlated and fused information about the learners.

The potential impact of such system is to allow continuous monitoring and support, where the immediate outcomes (feedback, report, learner models) can be subsequently validated by (i) iteratively adapting a game environment to match the individual learners' model and track the performance continuously, and (ii) evaluating the resulting intervention within a classroom setting, where teachers will be able to design their teaching strategy based on the analysis and insights.

The following sections further elaborate on these 4 components (fig. $2 b$ ) and the relevant studies that influence the ecosystem and the architecture of the proposed model. 


\subsection{Learning analytics platform: understanding learners/players using a SGs-tailored learning analytics approach}

The goal of learning analytics includes enabling teachers and schools to tailor educational opportunities to each student's level of need and ability. Learning analytics addresses and expands on the application of known methods and models to understand student learning and organizational learning systems. Unlike educational data mining, which emphasizes system-generated and automated responses to students, learning analytics enables human tailoring of responses, such as through adapting instructional content, intervening with at-risk students, and providing feedback [29]. With this perspective, existing tools, such as the Games and LEarning ANalytics for Educational Research (GLEANER, see fig. 2) has been implemented to support the tracking of learners and analyze their in-game activities. GLEANER, developed as part of the R\&D activity of the EU-Funded Games and Learning Alliance (GALA) network, represent both an abstract framework and an implementation to support the Learning Analytics approach applied to educational videogames [30].

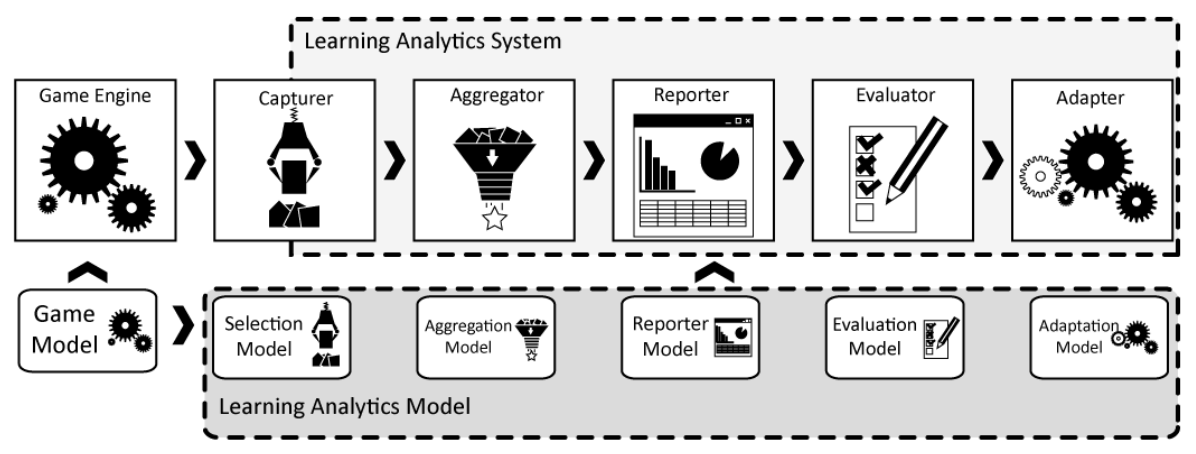

Figure 2. Main components of GLEANER, and their relationship with the game engine- from capturing and measuring data to reporting the analysis and adapting the existing environment to respond.

GLEANER is composed of a Learning Analytics Model (LAM) that defines all the information required for every step, and a Learning Analytics System (LAS) that comprises all the processing power required by the model (see Fig. 2). The abstract model defines the traces and data points to be collected, as well as an API to transmit the traces to a Learning Analytics System and how these data should be processed (see fig. 2).

The framework also provides a dashboard for instructors, in which it is possible to follow the flow of traces in real time, and therefore provide support for the instructor in his teaching. Based on the first results concerning the application of the framework and the use of the learning analytic tools, the model has evolved into a two-tier system [24].

The LAS component is created as a service, that can be remotely located, and which collects the traces generated by the game. The game pushes information to the server according to a specific API, and the instructors can also access the server separately to follow the updates.

The implementation of the service and the communication is structured around the following steps: 1. The game engine reports user interactions to the capturer module, filtering data according to the selection model.

2. The aggregator filters the received data and may aggregate and transform sets of low-level traces into higher lever traces according to the aggregation model. This allows the instructor to extract meaningful information of the student actions, based analysis of the students' game-play interactions that can be used in the later phases of the analysis.

3. The reporter module presents data and statistics in a human-friendly visualization, according to the instructions in the reporter model. This allows the instructor to get a summary of the students' performance levels at a glance, at individual student's level or at Course Level. This may also be used to allow students to compare their progress with their peers

4. The evaluator module can perform automatic assessment of the learning experience, checking the collected traces against a list of learning goals as described in the evaluation model. The objective is to predict the knowledge gain (or potential lack of).

5. The adapter module can use the information reported by the previous module to trigger changes in the state of the game, according to an adaptation model. 
6. These technical modules, along with their driving abstract models, are the base for the scaffolded approach presented in this work.

\subsection{Stealth and in-game measures: capturing meaningful interactions}

Meaningful interactions within the game are indeed the core of a learning experience. Meaningful in this case refers to interaction and engagement with contents that result in learning. This suggests an alternative approach to assessment based on tracking and analyzing the interactions during the learning experience, rather than using a separate instrument. The engagement with the learning content can be tracked using in-game measurement that enables the identification of the exploratory path taken during game-play and the specific content accessed by the players. Recorded information can be the number of times tutorials and hints are accessed, the amount of time spent on problemsolving mini games, the scores based on problems solved.

Based on the on-going studies on the GLEANER framework [31], we have identified that it is essential to establish which generic and specific data must be captured for an effective evaluation of educational games, depending on the individual characteristics of each simulation and game (field, scope, interaction mode, deployment device) and having a more general nature. The study of the types of in-game traces that may be used when applying Learning Analytics techniques to SGs was based on literature reviews and game analyses. Three main types of traces have been identified.

Generic traces are those that may be gathered from any SGs, regardless of the specific type [32]. However, the assessment value of such traces is limited by their generic nature. In the other extreme, we can define game-level traces, designed to track specific in-game actions relevant in a particular game design. Such traces can convey more information than generic traces, and be more useful both for assessment and evaluation. Such traces will require automatic means of analysis and reasoning for game-specific traces and this will imply that the application has to be scaled with respect to specific metrics and KPIs.

As a balance, genre-level traces offer a middle ground, allowing the effort to focus on specific traces that can be applied across a variety of games. An example would be the implementation of GLEANER on the eAdventure game platform [32].

The definition of game-specific (or genre-specific traces) is the key to understanding how the students are interacting with the game. GLEANER has now been used in several case studies [24, 33]. Based upon the experience, the model has evolved into a two-tier system allowing the construction of game-level and genre-level traces as aggregations of low-level traces [24].

In order to assess the activity in a video game, both at a general level, as well as at the individual participant level, game flow traces (consisting of game start, end and quit) provide information on whether the students have played it or if they have finished it. Many educational games have some sort of narrative structure with different phases and sub goals, thus it is also necessary to track the start and the end of phases. This is realized with phase change traces [24].

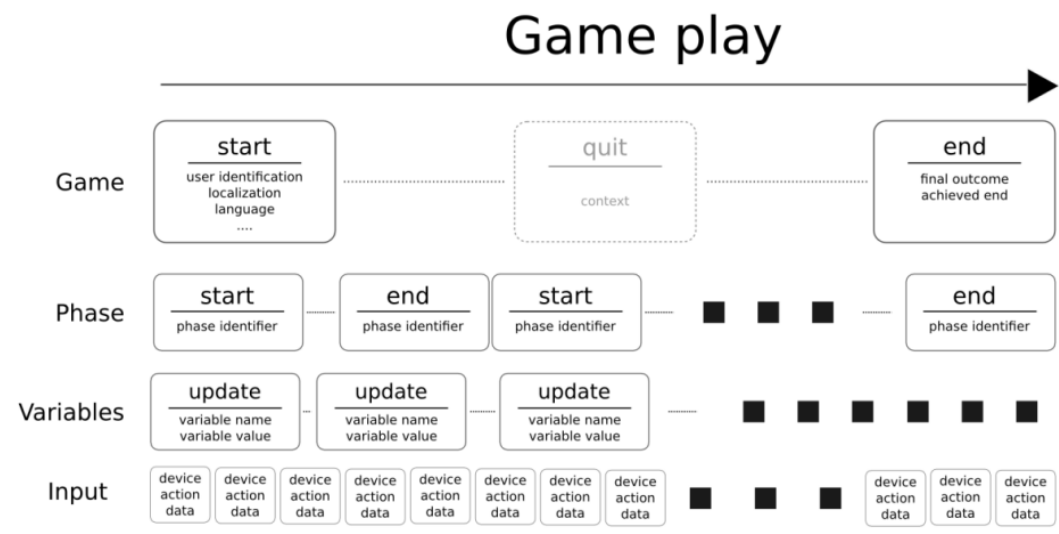

Figure 3. Diagram with all the traces of our "universal" set, with the data associated to every of them, ordered by frequency during the gameplay

In addition, all games use variables to keep some sort of game state. Depending on game play, learning objectives etc., the relevance of the different variables for LA varies, but typically variables to monitor are scores or attempts used to complete the game. By monitoring the evolution of relevant variables over time, we can observe intermediate states the player goes through and a posterior 
analysis can use final values for relevant variables to define a heuristic for player performance in the game. In addition, the model enables the collection of input traces, like the single clicks etc. This information can be transmitted to a back-end and stored for latter processing, in those cases where this is relevant for analyzing whether the learning goal are achieved or to what extent they are achieved.

Fig. 3 shows how the different traces and data related to each other throughout the game play and based on the frequency. Based upon the aforementioned traces aggregated traces can be defined. More details can be found in [24].

Section 4.2 will describe some case studies and the associated results.

\subsection{Mapping pedagogical patterns to game mechanics: theoretical constructs to game and learning interaction}

Tracking learner interaction during game play provides vital information that will allow different learning styles and conceptions of, and approaches to teaching using technology (see for example [34] to be analyzed as well as game play and content to be adapted. As described above, this operation is performed in GLEANER through the definition of a report model and an evaluation model that drive the transformation of the raw tracking data into reports and assessments. However, configuring these models to provide good insights into the learning process requires a deep understanding of how learning happens when the user interacts with the game.

Pedagogical requites dictate that in-game interactions pertaining to learning has to be driven by extracting the relationship between games and learning mechanics at the abstract and concrete level; the fundamentals behind what links a game design pattern to a pedagogical pattern. From a pedagogical perspective it is difficult to dissociate game mechanics (GMs) from educational components at the implementation level [35].

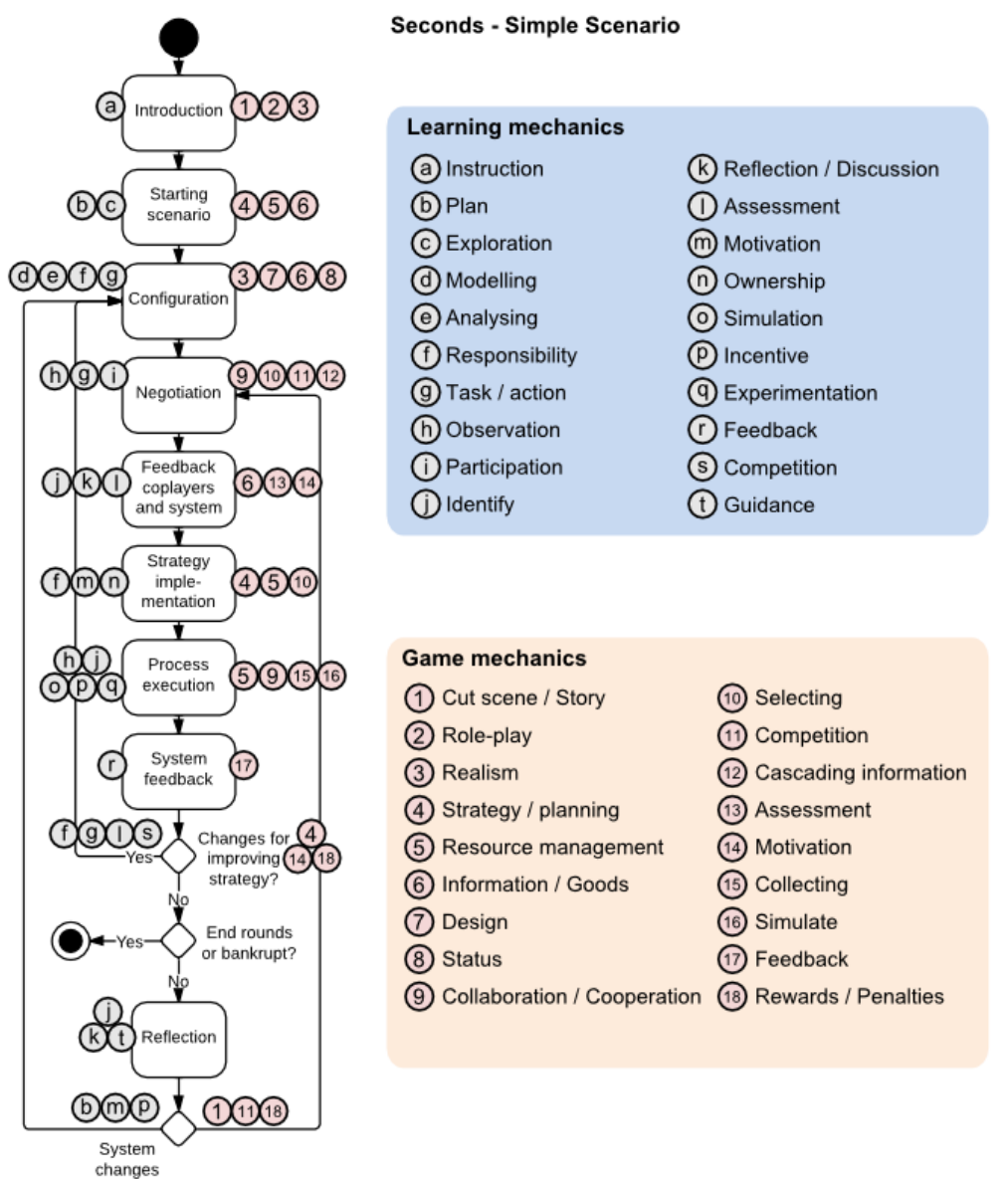

Figure 4. LM-GM mapping of Seconds [40]

In order to identify the influence and describe the relation between gaming mechanisms to mechanisms of some pedagogical practice attributed with the game, a learning mechanics and game mechanics framework (LM-GM) was developed [36, 37]. The LM-GM framework has been used by 
[23] to validate the relationship between the learning mechanics and game mechanics implemented in the PREPARe game, which has undergone a cluster randomized control trial that concluded positive learning outcomes. The LM-GM purpose-processing methodology has also been used to characterize the SG narrative-pedagogical design elements [38, 39].

In the case study presented here, pedagogical elements are considered abstract interfaces while game elements are deemed as a concrete interface of SGs. The example below shows the LM-GM mapping for a simple scenario of a multi-user game used for decision making [40] to illustrate the principle of scaffolding and personalized learning (Fig. 4).

For example, in the phase "configuration" the degree of freedom of design possibilities is driven by the game mechanics that provided information interactively as well as the tasks (via gaming levels) to elicit learners existing knowledge. The learning mechanics of modelling and analysis fosters the pedagogical practice of generating artefacts and hypothesizing the relationships between learning and the instructional moves they make. These are scaffold with game mechanics that simulate their decisions and inform about their status of gameplay. Artefacts can include learners' responses to ingame prompts, conversations, assessments, etc. that allow the SG to track and trace decision making competences from the first gaming level. Subsequent game levels would use the results achieved in the game play to deliver follow-on learning content.

This provides a clear relationship definition for the LAM in fig. 4 to track and process data. The LMGM mapping visualizes the configuration and flow of the game-based learning application and so allows quick identification of key engagement points in the game play. Based on similar principles, the LAM would be able to ascertain meaningful learning engagement during game play to be correctly tracked and assessed.

Mapping learning mechanics to game mechanics is crucial in order to inform the definition of ingame measures and to keep the balance in adaptation process. Previous studies $[38,41]$ have shown that without having a balancing mechanism between learning process and adaptation mechanisms, there will be uncertainty within the framework. Finally, no optimization is possible without mapping the real world learning process against artificial learning or game mechanics. In playing a game, learners exhibit, through their gaming activities and the choices they make, preferential interaction modes. However, the way they play and their preferences in terms of game-play are determined to a large extent by the game rules and game mechanics. As with education the associated outcome of games can be considered generic, i.e. the aim is to acquire knowledge, skills and attitude. Insofar, it is about the value of practical experience and the appreciation of developing personal and technical skills alongside knowledge. For this reason, understanding the motivations of the learner in SG games and their relationship towards pedagogical outcomes and the gaming experience is important to the scaffolding paradigm.

\subsection{User modelling, adaptive control and visual analytics: providing personalization}

The crucial importance of understanding mappings between game mechanics and learning mechanisms for achieving successful learning has been pointed out. Although these components are essential there is another dimension - individuality of learners. Individual learners' may have varying preferences with respect to engaging game play and game mechanics, they may respond differently to varying strategies for learning, they may have varying requirements with respect to cognitive aspects, and they may have varying prior knowledge; their requirements with respect to optimal learning varies. It is thus essential to understand the specific needs of individual learners' and to understand the relationship between preferences and content, in order to properly adapt and control game content to provide a more personalized experience. Adaptive web systems have investigated a range of approaches to user profiling. The most popular features modeled and used by adaptive web systems are user knowledge, interests, goals, background, individual traits, and context of work, while each individual adaptive system typically focuses on some of these factors [13].

User modelling refers to, in the context of games and learning, a process where multiple users are analyzed to distinguish categorizations of learners' with respect to e.g. learning behaviors, game play interaction, preference, content, which can be used for optimizing the learning process. More specifically, user models abstract the distinctive features of a user, so that it can be continuously updated by the game-based environment while giving input to a personalization engine, which adapts the contents and their provision modalities to the elicited requirements. Mathematics Navigator [42] for instance, applies Teachable Agents in recording and analyzing learners' knowledge and then optimizing the future learning path to override the difficulties in learning. Teachable Agents can also be used to decrease Information Overload [43] and so adapt the content to the learner's needs. User 
modelling methods have been widely used in the context of Intelligent Tutoring Systems, Adaptive Hypermedia Systems, which can be adapted to support user modelling in games for learning in conjunction with the GLEANER framework (fig. 3). In particular, the aggregated and refined outputs of the evaluator module are potentially a useful starting point for adaptation decisions inside the game.

The proposed ecosystem needs to engage and support stakeholders, teachers, students, school administrators and regional and state bodies by analyzing and exploiting large datasets. In order to provide interactive, personalized and immersive interfaces to deal with this data, as well as allowing a continuous adaptation of the process by the teachers, visual analytics (VA) can be used as a design framework. VA is defined as analytical reasoning supported by highly interactive visual interfaces [44] and it strives to facilitate the analytical reasoning process by creating software that maximizes the human capacity to perceive, understand, and learn from large, complex and dynamic data and situations.

Establishing the analytical reasoning process that needs to be supported for the different actors involved in the ecosystem proposed is essential to successfully develop the activities described by GLEANER, especially the reporter module and abstract model, that provides on one hand a set of predefined but customizable reporting views, but also provides extension points to add new modules to provide tailored views. VA advocates for the design of transparent user modelling and adaptation modules, guided in our case, by the teachers. Moreover, it is important to provide a user-friendly infrastructure - personalized dashboards to provide feedback, summaries, actionable steps, visualizations of the learning processes, statistics about the examination outcomes, etc. The system allows key players, such as the teachers and administrators, to be involved in the adaptation of the content based on the analysis of the learners' progress.

The use of VA and its benefits to support e-learning has recently been suggested by, for example [45]. Even if the application of VA principles to e-learning is considerably new, there are several examples in the literature that show the advantages of using interactive visual environments for learning through, for example, graphic organizers, such as mind or conceptual maps, or more advanced tools that permit the visual management of e-learning contents, e.g. Classroom BRIDGE, ENCCON, TM4L, etc. (see a more extensive review in [45]). Visualization can be also used to represent the learning process, its dynamics and interactions between students and groups. An example of the latter is CourseVis, presented in [46], where several $2 \mathrm{D}$ and $3 \mathrm{D}$ visualizations are provided for helping the instructors to form a mental model of their class and provide appropriate help.

\section{The conceptual ecosystem: towards a large-scale deployment}

The full value of these information technology tools cannot be reached unless they are seamlessly integrated within the ecosystem they are implemented in and the data they generate is available to wide audiences [47]. On a larger-scale, such technology-scaffold architecture could potentially support and benefit an ecosystem of learners, teachers, administrators, learning designers and game developers.

\subsection{The impact of the ecosystem approach}

Valuable analysis of learners within an educational context will guide teaching and learning strategy, resource management, adaptive game-based learning design and learning content development. Figure 5 illustrates an ecosystem aiming to support the capturing and reasoning of large-scale educational data from engaging sources to better understand learners' knowledge, assess their progress and provide actionable feedback, which will be relevant to the stakeholders involved.

Participatory approach is of key importance to the teaching and learning ecosystem, where the metrics and performance indicators specific to the stakeholders can be specified and included in the design of the intelligent learning environment [48]. 


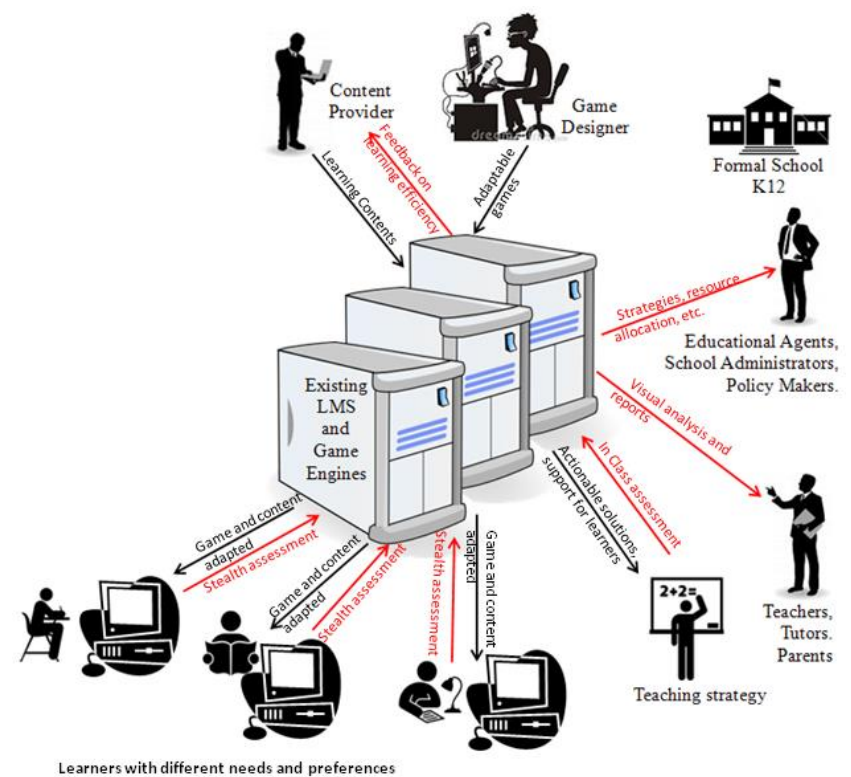

Figure 5. A conceptual ecosystem

Non-scientific users, such as learners, teachers, parents, school administrators and state/regional bodies, could be offered support and they will be able to engage with and exploit large datasets. Learners and teachers/instructors will obtain a better perspective of the educational process and its results based on the data collected and analyzed for a specific course/module. This will help individual learners to reach their learning goals and the teachers/instructors to provide the right support to their pupils/trainees. For example, MathElements, a game targeting pre-school children, teachers and parents, use learning analytics [43] to gain insights on learner's strengths and weaknesses, in order to support teacher and/or parent in instructing the learner. The Monster Manor game involves parents and clinicians aiming to motivate children with Type 1 Diabetes to check their blood sugar regularly [49]. Parents are able to monitor their child's progress and encourage positive and healthy behavior. The sustainability game helps students to raise awareness on sustainability issues for public spaces [50]. Teachers are able to provide feedback while students are playing on different quests and try to steer learning in relation to intended learning outcomes.

Predictive models and success/failure patterns can be correlated in the analyzed data based on the specific measures and metrics, which can be used to design actionable solutions to overcome the weakness of the teaching and learning strategy and resource allocation on the institutional level. For example, [50] studied the relation between learning outcomes, speed of interaction and nature of misunderstandings. Based on these parameters, they have suggested a simple adaptation algorithm on predicting the optimal learning zone. By analyzing individual learning processes, Math Elements also helps to identify topics in the curriculum that needs additional emphasis [51]. The ClassMATE framework [52] introduces a pervasive system that assists in a non-obstructive way the students during learning activities both at school and at home. ClassMATE supports collaborative tasks, application migration, service personalization and personalization of semantically discovered content to each individual learner's needs. Its main advantage is that every decision made and action taken is driven by contextual information (who, when, where, what) towards facilitating end user interaction (student or teacher).

The ecosystem should also support adaptivity, where the game-based learning environment can be adapted to support specific learner profiling which will allow longitudinal studies on how learning experience evolve with time and with respect to the change of teaching and learning strategy to respond to the learners' specific needs.

Bringing together SGs and Learning Analytics to facilitate the understanding and support of learners could offer an intelligent and interoperable learning framework capitalizing on learning analytics tools that can be implemented and integrated onto a LMS, a game engine or a game editor to provide a wide range of scaffolded support for learners and teachers/instructors within a formal setting. However, there are several challenges on recording big data on learning, privacy being one of the key issues. In terms of deployment within a formal setting, the context of deployment has to consider the existing infrastructure and the needs of the beneficiaries. 


\subsection{GLEANER case studies in support of the ecosystem approach}

Among the major advantages of GLEANER we highlight the following: it is open source, it offers an abstract framework as well as a reference implementation for a GLEANER trace aggregation server and a reference implementation for game clients (see section 3 and [24, 33]). This allows different game vendors and different LA systems to exchange data, which is a pre-requisite in order to realize the conceptual ecosystem outlined in section 4.1.

In order to be able to personalize the teaching, it is necessary that during game play the teacher is able to identify the challenges as well as the progress of each individual player. Currently this need and the lack of suitable LA tools often leads to very small classes as well as to a wide use of postgame reflection phases and debriefing session. However, GLEANER provides a dashboard that allows teachers to follow the flow of traces in real time. The objective of this tool is to help the instructor track the development of a gameplay session, with special focus on identifying which students are having issues with the game. I.e. the teacher is not any more dependent of his observation and experience as a teacher and facilitator, but he gets support from the analysis carried out during game play. This enables a more personalized feedback during game play and also allows larger classes, thus also contribute to the creation of an ecosystem.

In addition, in order to allow scaffolding at an individual level, it is necessary to be able to track the individual performance. The following case studies just briefly outline the possibilities GLEANER has. Complete case descriptions are to be found in [33].

Lost in Space $<X M L>$ is a puzzle game designed to learn basic XML and DTD concepts. The game was divided in phases of incremental difficulty. In each phase, students must lead a spaceship on a grid board from an origin point to a wormhole. They must type actual XML snippets (complying with a DTD that defines spaceship actions) that are then sent to an interpreter that translates the snippets into in-game actions that guide the spaceship. The case study focused on the runtime assessment and the effect of using LA in a live (game-based) classroom activity. It also covered using games instead of exams, by setting a number of in-game conditions and achievements that would allow a student to "pass". 37 students participated in this case study and three assessment conditions were defined to demonstrate the use of games as an evaluation tool, based on the in-game score, the percentage of valid XML snippets generated and the time required to complete the game. A runtime dashboard was setup and displayed on the instructors' computer, allowing him to track the progress of the students and detect wrong fragments of code.

The session was an open test of the feasibility of the approach and the usefulness of the reporting tools and the results showed that $94 \%$ of the students completed the game successfully, $81 \%$ of students achieved scores over 1000 . However, only $24 \%$ of the students completed the game in less than 30 minutes. However, in relation with the concept of scaffolding, the use of the runtime dashboard and its possibilities are of more interest.

It allowed the instructor to monitor the students' progress and to identify the individual differences (important to keep the students engaged, motivated and in flow) as well as to identify common points of difficulty (i.e to identify such elements where many students had to retry before passing). In this case, this information may indicate that the teacher needs to reinforce the meaning of the element or, indeed, that the game is not accurate enough, so that the students do not know what to do. The latter would require re-design, whereas the former helps the instructor to identify where the students still need more support and instruction.

In addition, these benefits are not only possible at group level, but specific individual challenges can also be identified, supporting this scaffolded approach. In this case study, for example, the instructor detected that a specific student was continuously sending fragments of DTD code (rather than XML code) suggesting a major misconception about the game and the technologies being studied. The instructor then approached this student and offered a clarification on the difference. The student rapidly acknowledged the misconception and then increased her performance significantly for the rest of the session. In this case study no formal approach was used. Actually, the instructor could carry out his observation as he used to, but now he had more support in monitoring the progress and providing personalized support. Since the instructor can apply all his knowledge and experience and since it supports his way of carrying out his work, it will be easier to implement in the daily operation. A second case study intended to use GLEANER as an analysis tool for understanding how students were learning by responding to in-game questions and displaying the progress through game play. 355 students from 9 different Spanish schools participated in the case study. The game in use was "The Foolish Lady" designed to motivate youngsters on classical theatre play [33]. Using the generic traces designed to track changes in specific in-game attributes, it was possible to offer the instructor an assortment of possible analyses on how students progressed through the game, and how they 
replied to in-game questions and mini quizzes. Using the generic traces designed to track changes in specific in-game attributes, it was possible to offer the instructor an assortment of possible analyses on how students progressed through the game, and how they replied to in-game questions and mini quizzes. In addition, each individual response from each student was recorded, and detailed statistics collected on the frequency of each response. The study also looked at the potential for data aggregation, allowing stakeholders to get performance information with varying granularity, from a single student to a large set of schools. The picture below shows the aggregated the results (fig. 6).

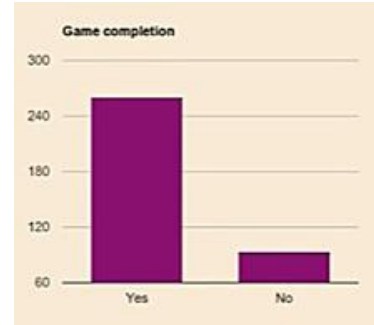

Figure 6. GLEANER case study results - The follish Lady" game

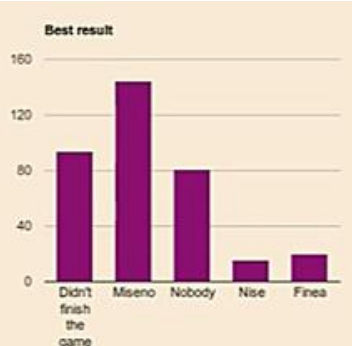

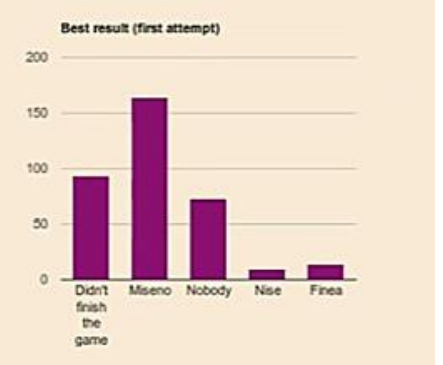

The data was structured and analyzed allowing different levels of aggregation. It was possible to visualize data for a single student, a specific gameplay session or all the schools in the region. The possibility of aggregating results as well as comparing results are important for being able to meet the increasing number of students and also in order to ensure that students receive the same education at different schools. In addition, since the setup is done centrally, it reduces the need for teachers of being able to deal with technical implementation. The teachers can focus more on the pedagogical interpretation of the results (here of course also at an individual level).

These case studies demonstrate the potential of LA tools to create an SG-driven ecosystem, where assessment outcomes are automatically generated and naturally feed the "inhabitants" of the ecosystem to serve different purposes: enhance student performance; enable teachers to adapt the learning experience in real time; enable parents understand the rationale behind grades; enable state/ regional bodies adapt the learning strategies to better accommodate a larger variety of students.

\section{Conclusions and future work}

Game-based solutions are widely being deployed in schools, universities and training institutions, and efforts are being made to create wide-applicable, scalable support analytic tools that enable effective performance monitoring, evidence-based feedback, and higher level personalization to address specific user needs of both learners and teachers/instructors.

Learning Analytics emphasizes on the measurement and data collection as activities that educational organizations and teachers need to undertake and understand, and focuses on the analysis and reporting of the data, which will subsequently allow actionable steps and measures to be designed to overcome potential issues or weaknesses [25]. This paper brings evidence to support the use of LA for scaffolding game based-learning and teaching experiences. The authors present a conceptual architecture and supporting ecosystem that reveal the potential of using LA in SGs as scaffolding tools for learning and teaching experiences. The four components of the model are described in section 3: (i) learning analytics platform, (ii) in-game and stealth measures, (iii) user modelling, adaptive control and visual analytics, and (iv) map of pedagogical patterns to game mechanics, while section 4 present the conceptual ecosystem and case studies that bring evidence to validate the proposed approach.

The proposed model presents an opportunity for future evaluation of how the analytics and adaptation affects the learning experience by comparing the adapted learning experience with a control group using a default non-adapted version of the teaching and learning strategy or of the same SG. The deployment evaluations will include empirical studies, such as cluster randomized control trials- with intervention and without intervention for both scenarios: game-based learning environment and the classroom teaching environment (pre and post surveys, questionnaires, analysis of in-game data, analysis of classroom performance, etc). Intervention in this case will be the scaffolded support based on the proposed model.

In addition to experimental or quasi-experimental impact analysis, the evaluation could adopt a mixed method approach to explore changes in other important dimensions that may result from the 
engagement with the ecosystem, including but not limited to: (i) Changes in teaching practices, including changes in subject-specific and cross-curricular pedagogic approaches; (ii) Changes in patterns of homework/schoolwork among students, (iii) Changes in school-level approaches to curriculum design and implementation; and (iv) Changes in perceptions and attitudes among students in relation to interests and aspirations.

This opportunity will potentially affect the design and deployment of education and training in the future. The vast amount of data that is being collected about students also has policy implications in addition to technical ones. The investigation of how the collection and analysis of this educational data fits current institutional policies as well as European guidelines and ethical procedures can also be carried out. The availability of this type of data in different countries presents a unique opportunity. Not only will it be possible to investigate differences between countries and more accurately map and translate qualifications between countries, it will also have the potential to reveal differences between educational institutions and countries in terms of effectiveness.

\section{Acknowledgements}

This work has been co-funded by the EU under the FP7, in the Games and Learning Alliance (GALA) Network of Excellence, Grant Agreement nr. 258169.

\section{References}

[1] http://europa.eu/legislation_summaries/education_training_youth/general_framework/ef0016_ en.htm [Accessed 2013 Sept 30]

[2] Silva, E., Measuring Skills for 21st-Century learning. The Phi Delta Kappan. 90(9), 630-634, 2009. http://dx.doi.org/10.1177/003172170909000905

[3] Ananiadou \& Claro, 21st Century Skills and Competencies for New Millennium Learners in OECD Countries, 2009. Available at: http://www.oecd-ilibrary.org/education/21st-centuryskills-and-competences-for-new-millennium-learners-in-oecd-countries_218525261154 [Last accessed 16 March 2013].

[4] Dede, C., Comparing frameworks for 21st Century Skills, 2009. Available at: http://dca1 to1.pbworks.com/f/21st+Century+-+will+our+students+be+prepared.pdf [Last Accessed 15 March 2013].

[5] Eurydice, Eurydice Report- Developing Key Competencies at School in Europe: Challenges and Opportunities for Policy, 2012. Available at: http://eacea.ec.europa.eu/education/eurydice/documents/thematic_reports/145EN.pdf [Last accessed 17 March 2013].

[6] Vygotsky, L. S., Mind in society: The development of higher mental processes (M. Cole, V.John-Steiner, S. Scribner, \& E. Soubeman, Eds.). Cambridge, MA Harvard University Press, 1978.

[7] Wood, D., Brunet; J. \& Ross, G., The role of tutoring in problem solving. Journal of Child Psychologyand Psychiatry and Allied Disciplines 17, 89-100, 1976. http://dx.doi.org/10.1111/j.1469-7610.1976.tb00381.x

[8] Kolb, D.A., Experiential learning: Experience as the source of learning and development. Prentice-Hall Englewood Cliffs, NJ, 1984.

[9] Coffield, F., Moseley, D., Hall, E \& Ecclestone, K., Learning styles and pedagogy in post-16 learning: a systematic and critical review, LSRC reference, Learning \& Skills Research Centre, London, 2004.

[10]Duval. E., Learning Analytics for Visualisation and Recommendation, in Proceedings of LAK11: 1st International Conference on Learning Analytics and Knowledge, 2011.

[11]Fournier, H., Kop, R, Hanan, S., The Value of Learning Analytics to Networked Learning on a Personal Learning Environment, 2011, Available from: http://nparc.cisti-icist.nrccnrc.gc.ca/npsi/ctrl?action=rtdoc\&an=18150452\&lang=en [Last accessed 8 September 2013]. Publications Archive Canada. Canada.

[12] Manouselis, N., Drachsler, H., Vuorikari, R., Hummel, H. \& Koper, R., Recommender Systems in Technology Enhanced Learning, in Recommender Systems Handbook, F. Ricci, Rokach, L, Shapira, B, Kantor, P, Ed., ed: Springer, US, pp. 387-415, 2011.

[13] Brusilovsky, P. and Millán, E., User models for adaptive hypermedia and adaptive educational systems. In: P. Brusilovsky, A. Kobsa and W. Neidl (eds.): The Adaptive Web: Methods and 
Strategies of Web Personalization. Lecture Notes in Computer Science, Vol. 4321, Berlin Heidelberg New York: Springer-Verlag, pp. 3-53, 2007. http://dx.doi.org/10.1007/978-3-54072079-9

[14]Heilman, M., Collins-Thompson, K., Callan, J., Eskenazi, M., Classroom success of an Intelligent Tutoring System for lexical practice and reading comprehension. Proceedings of Interspeech 2006. Pittsburgh, U.S.A, 2006.

[15] Siemens, G., Learning Analytics: Envisioning a Research Discipline and a Domain of Practice, in Learning Analytics and Knowledge 2012, Vancouver, Canada, 2012.

[16] Ali, L., Hatala, M., Gašević, D. \& Jovanović, E., A qualitative evaluation of evolution of a learning analytics tool. Computers \& Education 58(1), 470-489, 2012. http://dx.doi.org/10.1016/j.compedu.2011.08.030

[17] Dawson, S., Bakharia, A., \& Heathcate, E., SNAPP: Realising the affordance of real-time SNA within networked learning. In proceedings of the 7th International Conference on Networked Learning, Aalborg, Denmark, pp. 125-133, 2012.

[18] Buckingham Shum, S. \& Ferguson, R., Social Learning Analytics. Educational Technology \& Society 15(3), 3-26, 2012.

[19] http://www.nmc.org/pdf/2013-horizon-report-HE.pdf [Accessed 1 October 2013]

[20] Downes, S., Dell Cloud Services: Collaboration, Analytics and the LMS: A Conversation with Stephen Downes, 2010, Available at: http://campustechnology.com/newsletters/ctfocus/2010/10/collaboration_analytics_and-thelms_a-conversation-with-stephen-downes.aspx2010.[Last accessed 9 Sept 2013].

[21] Kop, R., The Design and Development of a Personal Learning Environment: Researching the learning experience. Presented at the European Distance and E-Learning Network, Valencia, Spain, 2010.

[22] Peters, V. a. M. \& Vissers, G. a. N., A Simple Classification Model for Debriefing Simulation $\begin{array}{lllll}\text { Games. } & \text { Simulation } \quad \& \quad \text { Gaming }\end{array}$ http://dx.doi.org/10.1177/1046878103253719

[23] Arnab, S., Brown, K., Clarke, S., Dunwell, I., Lim, T., Suttie, N., Louchart, S., Hendrix, M. \& de Freitas, S., The Development Approach of a Pedagogically-Driven Serious Game to support Relationship and Sex Education (RSE) within a classroom setting. Computers \& Education 69, 15-30, 2013. http://dx.doi.org/10.1016/j.compedu.2013.06.013

[24] Serrano-Laguna, A., Torrente, J., Moreno-Ger, P., Fernàndez-Manjòn, B., Application of Learning Analytics in educational videogames. Entertainment Computing. Vol. 5 (4), pp. 313 322, 2014, doi:10.1016/j.entcom.2014.02.003.

[25] Baalsrud Hauge, J, Berta, R, Fiucci, G., Manjon,B.F., Padron-Napoles,C.L., Westra, W. Nadolski, R. "Implications of Learning Analytics for Serious Game Design",ICALT, 2014, IEEE 14th International Conference on Advanced Learning Technologies (ICALT), pp. 230232, 2014, doi:10.1109/ICALT.2014.73

[26] Shute, V. J., Stealth Assessment in Computer-Based Games to Support Learning. In S. Tobias \& J. D. Fletcher (Eds.), Computer Games and Instruction. Information Age Publishers, pp. 503523, 2011.

[27] Bellotti, F., Kapralos,B., Lee,K. and Moreno-Ger, P."User Assessment in Serious Games and Technology-Enhanced Learning," Advances in Human-Computer Interaction, vol. 2013, Article ID 120791, 2 pages, 2013. doi:10.1155/2013/120791

[28] Ketamo, H. \& Kiili, K., Conceptual change takes time: Game based learning cannot be only supplementary amusement. Journal of Educational Multimedia and Hypermedia19(4), 399-419, 2010.

[29]Bienkowski, M., Feng, M. \& Means, B., Enhancing Teaching and Learning Through Educational Data Mining and Learning Analytics. An Issue Brief. U.S. Department of Education Office of Educational Technology, 2012.

[30] Serrano-Laguna, Á., Marchiori E. J. et al., A framework to improve evaluation in educational games. In Proceedings Proceedings of the IEEE Engineering Education Conference (EDUCON), Marrakesh, Morocco, 2011.

[31] Moreno-Ger, P., Manjón, B. F, Laguna, A. S., Ortiz, I. M., Kiilli, K., Ninaus, M., Kober, S. E., Wood, G., Neuper, C., Berta, R., van Oostendorp, H., Wouters, P., Veltkamp, R., Arnab, S., Imiruaye, O., Dunwell, I. \& Liarakopis, F., D2.3 Priority Area 1 Report, WP2 Research and Development, Games and Learning Alliance (GALA), 2013.

[32] Serrano-Laguna, A., GLAS: a framework to improve assesment in educational videogames (Master Thesis). Universidad Complutense de Madrid, 2012, Obtained from UCM e-Prints: http://eprints.ucm.es/16929/ 
[33] Arnab, S., Moreno-Ger, P., Lim, T., Ott, M., Stanescu, I., Kiili, K., Perttula A., Earp, J., Ninaus M., Louchart, M., Jessel, J.P., Orliac, C., WP2 Synthesis and Summary report. Deliverable n. 2.4, 2014, online: http://www.galanoe.eu/index.php?option=com_content $\&$ view $=$ article $\&$ id $=700 \&$ Itemid $=182 \&$ dir=JSROOT\%5CDeliverables\%5CYear+4LDEL_WP2+\%2B+TC+Reports

[34] Lameras, P., Levy, P, Paraskakis, I, Webber, S,. "Blended university teaching using virtual learning environments: conceptions and approaches." Instructional Science 40(1): 141-157, 2012. http://dx.doi.org/10.1007/s11251-011-9170-9

[35] Suttie, N., Louchart, S., Lim, T., Macvean, A., Westera, W., Brown, D., Djaouti, D., Introducing the "Serious Games Mechanics" A Theoretical Framework to Analyse Relationships Between "Game" and "Pedagogical Aspects" of Serious Games, Procedia Computer Science, 15: 314315, 2012. http://dx.doi.org/10.1016/j.procs.2012.10.091

[36]Lim, T., Louchart, S., Suttie, N., Ritchie, J., Aylett, R., Stanescu, I. A., Roceanu, I., MartinezOrtiz, I., \& Moreno-Ger, P., Strategies for Effective Digital Games Development and Implementation. In Y. Baek, \& N. Whitton (Eds.), Cases on Digital Game-Based Learning: Methods, Models, and Strategies (pp. 168-198). Hershey, PA: Information Science Reference, 2013.

[37]Lim, T., Louchart, S. \& Suttie, N., D2.2 TC2.1 Serious Games Mechanics Report, WP2 Research and Development, Games and Learning Alliance (GALA), 2012.

[38] Arnab, S., Lim, T., Carvalho, M. B., Bellotti, F., de Freitas, S., Louchart S., Suttie, N., Berta R., De Gloria, A. (in press). Mapping Learning and Game Mechanics for Serious Games Analysis, British Journal of Educational Technology, 5 JAN 2014, DOI: 10.1111/bjet.12113.

[39] Lim, T., Louchart, S., Suttie, N., Baalsrud Hauge, J., Stanescu, I. A., Ortiz, I. M., Moreno-Ger, P., Bellotti, F., Carvalho, M.B., Earp, J., Ott, M., Arnab, S., \& Berta, R., Narrative Serious Game Mechanics (NSGM)-Insights into the Narrative-Pedagogical Mechanism. In Games for Training, Education, Health and Sports (pp. 23-34). Springer International Publishing, 2014. http://dx.doi.org/10.4018/978-1-4666-2848-9.ch010

[40] Baalsrud Hauge, J. M., Stanescu, I.A., Carvalho, M. B., Lim, T., Louchart, S., Arnab, S., Stefan, A., Serious Game Mechanics and Opportunities for Reuse. In proceedings of the 11th International Scientific Conference eLearning and software for Education (eLSE 2015), Bucharest, Romania (accepted paper), 2015.

[41] Ketamo, H., Balancing adaptive content with agents: Modeling and reproducing group behavior as computational system. In proceedings of 6th International Conference on Web Information Systems and Technologies, WEBIST 2010, Valencia, Spain, 1, pp. 291-296, 2010.

[42]41. Ketamo, H., Alajääski, J. \& Kiili, K., Self-Organizing Learning Material on Teacher Education. In Proceedings of EdMedia, Honolulu, Hawaii, pp. 3658-3667, 2009.

[43] Ketamo, H., Managing Information Overload - Teachable Media Agents. In Proceedings of the 8th International Conference on Intellectual Capital, Knowledge Management \& Organisational Learning - ICICKM 2011. Bangkok, Thailand, pp. 301-308, 2011.

[44] Thomas, J.J. \& Cook, K.A., Illuminating the Path: The Research and Development Agenda for Visual Analytics. National Visualization and Analytics Ctr. IEEE Computer Society, Los Alametos, CA, 2005.

[45] Gómez Aguilar, D. A., Suárez Guerrero, C., Therón, R. \& García Peñalvo, F., Visual analytics to support e-learning In Ed. Mary Beth Rosson (pp. 207-228) IN-TECH: Advances in Learning Processes, 2010.

[46] Mazza, R. \& Dimitrova, V., Generation of graphical representations of student tracking data in course management systems. 9th International Conference on Information Visualisation, pp. 253-258, 2005.

[47] Bellotti, F., Berta, R., De Gloria, A., D'Ursi, A. and Fiore, V., A serious game model for cultural heritage. ACM J. Comput. Cult. Herit. 5, 4, 2012.

[48] Stanescu I.A., Stanescu, A.M., Moisescu, M., Sacala, I.S., Stefan, A. Baalsrud Hauge, J., Enabling Interoperability Between Serious Game and Virtual Engineering Ecosystems. Proceedings of the VES: Game Eco-Systems in Engineering, 34th Computers and Information in Engineering Conference (CIE), ASME 2014 International Design and Engineering Technical Conferences \& Computers and Information in Engineering Conference (IDETC/CIE 2014), August 17-20, 2014 in Buffalo, NY.

[49] Arnab, S., Hobby to habits. Public Service Review: Health and Social Care 35, 105-105, 2013.

[50] Lameras, P., Petridis, P, Dunwell, I, Hendrix, M, Arnab, S, de Freitas, S, Stewart, C., A Gamebased Approach for Raising Awareness on Sustainability Issues in Public Spaces. The Spring 
Servitization Conference: Servitization in the multi-organisation enterprise 20-21 May 2013 Aston Business School Birmigham, UK, 2013.

[51] Ketamo, H., Sharing Behaviors in Games and Social Media. International Journal of Applied Mathematics and Informatics 5(1), 224-232, 2011.

[52] Leonidis, A., Margetis, G., Antona, M., Stephanidis, C., ClassMATE: Enabling Ambient Intelligence in the Classroom. World Academy of Science, Engineering and Technology, 66, pp. $594-598,2010$. 